\title{
OUTCOME OF PRESUMPTIVE VERSUS RAPID DIAGNOSTIC TESTS-BASED MANAGEMENT OF CHILDHOOD MALARIA - PNEUMONIA OVERLAP IN URBAN NIGERIA: A PILOT QUASI- EXPERIMENTAL STUDY
}

\author{
Kingsley N. UKWAJA (MBBS) ${ }^{* a, b}$, Olufemi B. AINA (MBBS) $)^{\mathrm{a}}$, Ademola A. TALABI (MBBS)
}

\begin{abstract}
BACKGROUND: Symptoms of malaria and pneumonia overlap in African under-five children and the integrated management of childhood illness strategy require that such children be managed presumptively with both antibiotics and antimalarials. A 2003 WHO expert meeting recommended the evaluation of malaria rapid diagnostic test in the management of children with this overlap, but this has not been evaluated. Therefore, the objective of this study was to compare the clinical outcome of presumptive versus malaria rapid diagnostic test based management of childhood malaria-pneumonia overlap in Nigeria.

METHODS: A pilot quasi-experimental study was conducted November 2009 through February 2010 in an urban comprehensive health centre in Ogun, South-Western Nigeria. First, 50 children with malaria-pneumonia symptom overlap were consecutively enrolled and treated presumptively with antibiotics and antimalarials irrespective of malaria test result (control arm).Then, another 50 eligible children were enrolled and treated with antibiotics with/out antimalarials based on rapid diagnostic test result (intervention arm). Primary endpoint: clinical cure at day-5. The data were analyzed using Epi Info version 3.4.1.

RESULTS: The intervention and control arms did not differ significantly regarding patient demographic and clinical characteristics. Clinical cure rate was slightly higher in children managed presumptively 49 (98\%) than those managed rapid diagnostic test -based $47(94 \%)(P=0.31)$. However, rapid diagnostic test -based treated children had lower risk of receiving antimalarials compared to those treated presumptively (48\% vs. 100\%), $(P=$ $<0.001$; relative risk 2.08, CI 1.56 to 2.78). No death or severe complications were recorded in either group at day-5 follow-up.

CONCLUSION: Outcome of rapid diagnostic test-based treatment is not inferior to presumptive management in children with malaria-pneumonia symptom overlap. More extensive studies with larger sample sizes are needed.
\end{abstract}

KEYW ORDS: Malaria management, malaria- pneumonia overlap, rapid diagnostic tests, safety, Nigeria

\section{INTRODUCTION}

Because of the symptom overlap between malaria and pneumonia in African children, the World Health Organization (WHO) Integrated Management of Childhood Illness (IMCI) strategy requires that such children be managed presumptively with both antibiotics and antimalarials $(1,2)$. The widespread adoption of artemisinin based combination therapy antimalarials in low-resource settings and the availability of malaria rapid diagnostic tests (RDTs) have led to the modification of the malaria treatment policy by the WHO. The policy recommended malarial treatment only after parasitological confirmation-this recommendation had initially been restricted to older children and adults, but in the guideline's second edition (released $2^{\text {nd }}$ March 2010) the RDT-based policy has been extended to younger children (3).

Although the accuracy of RDTs for malaria infection has been demonstrated (4), there is limited evidence for the safety of a test -based strategy for children below five years $(5,6)$. Also, though the safety of not treating RDT negative children has been confirmed, such studies were conducted mainly in the context of childhood fevers (7). In 2003, a WHO consultative meeting on childhood pneumonia recommended that studies to improve the specificity of clinical overlap for malaria and pneumonia diagnosis should be undertaken, and rapid diagnostic tests for malaria should be studied to differentiate malaria from

a. Comprehensive Health Centre, Oke-llewo, Abeokuta, Ogun state, Nigeria

b. Department of Internal Medicine, Ebonyi State University Teaching Hospital, Abakaliki, Ebonyi State, Nigeria

*Corresponding au thor: Dr Ukwaja Kingsley N, MD; P.O.Box 06, Abakaliki, Ebonyi State, Nigeria. E-mail: ukwajakingsley@yahoo.co.uk 
pneumonia (8). We found no study that evaluated the use of RDT in the treatment of childhood malaria pneumonia overlap. Therefore, this study was conducted to compare the outcome of presumptively treated children having this overlap with antibiotics and antimalarials irrespective of malaria test result versus children treated with antibiotics with/out antimalarials based on RDT result in an area of high malaria trans mission.

\section{PATIENTS AND METHODS}

This was a pilot quasi-experimental study conducted November 2009 through February 2010. First, November through December 2009, fifty children with malaria-pneumonia symptom overlap were consecutively enrolled and treated presumptively with antibiotics and antimalarials irrespective of malaria test results according to national/ WHO/UNICEF guidelines (control arm). Then, January through February 2010, another 50 eligible children were consecutively enrolled and treated with antibiotics with/out antimalarials based on rapid diagnostic test result (intervention arm). The primary endpoint was clinical cure at day-5. Secondary endpoints were frequency of clinic re-attendance; persistence of presenting problems at follow-up, and proportion of unnecess ary antima larial treatment.

The study was performed in an urban comprehensive health centre in Abeokuta-south district, Ogun state, South-western Nigeria. The health centre is located in the rain forest belt of Nigeria, an area with perennial malaria trans mis sion.

Inclusion criteria were; age 2 - 59months, clinical (IMCI) malaria (defined as axillary temperature of $\geq 37.5^{\circ} \mathrm{C}$ or a history of fever within 48hours of presentation) with clin ical (IMCI) pneumonia (defined as a presence/history of cough and difficult breathing along with clinician counting of respiratory rate above IMCI cut-offs - a rate of 40 per-minute for children aged 12-59months and above 50 per-minute for children 2-12months), living within 3 kilometers of the health centre, and provision of informed consent. Exclusion criteria were; severe clinical state requiring urgent attention; presence of co-morbidities like otitis media, persistent vomiting, diarrhea etc.

The children were examined by a study clinician and their axillary temperature was measured. Capillary blood was obtained and malaria rapid diagnostic test was performed by a laboratory technician using Paracheck Pf (Orchid Biomedical Systems, India).In the control arm, all the study children received oral antimalarial (artesunate - amodiaquine combination antimalarials) and antibiotic (amoxicillin) irrespective of RDT result. And in the intervention arm, children with a positive RDT result received both antimalarial and antibiotics while those with a negative RDT result received antibiotic only. The artesunate - amodiaquine combination antimalarials used was a 3-day regimen pre-packaged for children 2-11 months or 12-59 months. The oral amoxicillin (antibiotics) used was prescribed at $50 \mathrm{mg} / \mathrm{kg} /$ day in two divided doses for five days.

All data were recorded in a standard IMCI forms and a follow-up was performed for all patients at day 5 . Patients were instructed to return for as sessment at any time if the symptoms deteriorated during or after the day-5 follow-up period. Patients who failed to visit the hospital were actively followed - up on day -6 .

Data were double-entered and analyzed with Epi Info (CDC Atlanta, version 3.4.1). Continuous variables were expressed as median ( \pm standard deviation) and categorical variables summarized using percentiles. Group comparisons were made (where appropriate) using the Wilcoxon two-sample test and uncorrected chi-square test, and their corresponding P-values were used for statistical inference.

Before data collection, the Research Committee Primary Health Care and Disease Control department of Abeokuta-south local government area, Ogun state, Nigeria read and approved the study protocol. Verbal informed consent was obtained from parents/legal guardian of enrolled child ren.

\section{RES ULTS}

The children in the control and intervention arms did not differ significantly in their demographic and clinical characteristics (Table 1). Overall, only $46 \%$ (95\% confidence interval (CI); 36\% - 56\%) of the study children had a positive malaria test using RDT. A cure rate of $98 \%$ was recorded in the control arm versus $94 \%$ in the intervention $\operatorname{arm}(\mathrm{P}=0.31)$ (Table 2). 
Table 1: Comparis on of two treatment groups at enrollment in Abeokuta-south district, Ogun Nov. 2009 - Feb. 2010.

\begin{tabular}{|c|c|c|c|c|}
\hline Variable & $\begin{array}{l}\text { Control arm } \\
\text { (Presumpti ve) }\end{array}$ & $\begin{array}{l}\text { Inter vention arm } \\
\text { (RDT-based) }\end{array}$ & $\begin{array}{l}* \text { Wilcoxon/ } \\
\text { Chi-s quare }\end{array}$ & $\mathbf{P}$ - value \\
\hline Total enrolled & 50 & 50 & & \\
\hline \multicolumn{5}{|l|}{ Age (months) } \\
\hline$\leq 12$ & $33(66 \%)$ & $31(62 \%)$ & 0.17 & 0.68 \\
\hline$>12$ & $17(34 \%)$ & $19(38 \%)$ & & \\
\hline Median $( \pm$ SD) & $12( \pm 11.2)$ & $12( \pm 9.4)$ & $* 1.6$ & 0.2 \\
\hline \multicolumn{5}{|l|}{ Gender } \\
\hline Male & $27(54 \%)$ & $25(50 \%)$ & 0.16 & 0.69 \\
\hline Female & $23(46 \%)$ & $25(50 \%)$ & & \\
\hline \multicolumn{5}{|l|}{ Axillary temperature } \\
\hline Range & $36.5-39.2$ & $36.7-39.8$ & & \\
\hline Median $( \pm$ SD $)$ & $37.9( \pm 0.79)$ & $38( \pm 0.6)$ & $* 0.8$ & 0.77 \\
\hline \multicolumn{5}{|c|}{ Malaria rapid diagnostic test } \\
\hline Positive & $22(44 \%)$ & $24(48 \%)$ & 0.16 & 0.69 \\
\hline Negative & $28(56 \%)$ & $26(52 \%)$ & & \\
\hline
\end{tabular}

No death or severe complications were recorded in either group at day-5 follow-up. Median fever clearance time was lower in the control arm $(2 \pm 0.65$ days $)$ than in the intervention arm $(3 \pm 0.7$ days $)(\mathrm{P}=$ $<0.001)$. However, rapid diagnostic test -based treated children had lower risk of receiving antimalarials compared to those treated presumptively $(48 \% \mathrm{vs}$.
$100 \%),(\mathrm{P}=<0.001$; relative risk 2.08 , CI 1.56 to 2.78). Three children in the intervention arm versus one child in the control arm had persistence of symptoms during follow-up $(P=0.31)$. A repeat RDT for all of them was negative and they were all referred to a tertiary hospital (Table 2).

Table 2: Clinical outcome at follow-up (day - 5) in the two groups in Abeokuta-South district, Ogun, Nov. 2009 - Feb. 2010 .

\begin{tabular}{|c|c|c|c|c|}
\hline Variable & $\begin{array}{l}\text { Control arm } \\
\text { (Presumpti ve) }\end{array}$ & $\begin{array}{l}\text { Inter vention arm } \\
\text { (RDT-based) }\end{array}$ & $\begin{array}{l}\text { *Wilcoxon/ } \\
\text { Chi-square }\end{array}$ & $\mathbf{P}$ - value \\
\hline Clin ical cure & $49 / 50(98 \%)$ & $47 / 50(94 \%)$ & 1.04 & 0.31 \\
\hline Early clinic re-attendance & $3 / 50(6 \%)$ & $9 / 50(18 \%)$ & 3.4 & 0.065 \\
\hline \multicolumn{5}{|l|}{ Fever clearance time (days) } \\
\hline Range & $2-4$ & $2-5$ & & \\
\hline Median $( \pm S D)$ & $2( \pm 0.65)$ & $3( \pm 0.7)$ & $* 32.1$ & $<0.001$ \\
\hline Proportion given antimalarial & $50 / 50(100 \%)$ & $24 / 50(48 \%)$ & 35.1 & $<0.001$ \\
\hline Persistence of symptoms & $1 / 50(2 \%)$ & $3 / 50(6 \%)$ & 1.04 & 0.31 \\
\hline
\end{tabular}

\section{DISCUSS ION}

This study showed that more than half of under-five children with malaria - pneumonia overlap do not have malaria parasitaemia using RDT. Thus, more than half of all antimalarials prescribed presumptively to children with overlap were unnecessary. This finding agrees with that of a Tanzanian study which showed that $43 \%$ of the fever in children was caused by acute respiratory infection (ARI) (9). However, it is not consistent with a recent Cameroonian study which showed that ARI accounted for $25.5 \%$ of fevers in children $0-2$ years old (10). These observations suggest that presumptive management of malaria pneumonia overlap in children is associated with antima larial drug wastage. Also, it risks the children to be exposed to unnecessary adverse drug reactions like; gastro-intestinal disturbances, neutropenia, hepatotoxicity and neurotoxicity which may be associated with artemisin in - based therapies (11). 
After decades of presumptive treatment strategies, the restriction of antimalarials to parasitologicallyconfirmed cases is necessarily difficult (12). Although recent studies have demonstrated the safety of a RDTbased strategy for child ren below five years $(5,6,10$, 12), such studies were conducted in the context of fevers in children. We demonstrate that the clinical cure rate of children with malaria - pneumonia overlap treated based on RDT results is not inferior to those managed presumptively. Thus, RDT-based strategy may be considered safe in the management of children with malaria - pneu monia overlap.

A major drawback of the RDT - based strategy in the management of fevers in low resource settings is healthcare providers' failure to adhere to not prescribing antimalarials to children with a negative test $(6,10,12)$. This may be experienced more so in children who had malaria - pneumonia overlap because such children will be brought to the hospital in a clinical state enough to cause anxiety amongst their caretakers for they might have attempted home management of the fever as malaria. Although adopting RDT with targeted training of health professionals cannot be assumed to translate adherence to treatment based on test results, strenuous efforts will be needed to understand, and change, existing clinical behavior where healthcare professionals have been used to ignoring negative malaria tests (12).

Children managed based on RDT had a higher median fever clearance time, and re-attended clinic more during follow-up compared to those managed presumptively. This is not consistent with observations of the RDT strategy made in the context of fevers in children $(6,10)$. The reason/s why a higher proportion of RDT-based managed children had a higher median fever clearance time compared to those managed presumptively are not clear. The implication of late fever clearance in patient treated RDT-based is that caretakers of such children are more likely to re-attend the clinic early and healthcare workers may be tempted to prescribe antimalarials despite a negative RDT result.

This is a pilot study; therefore, the findings are at best preliminary and need to be interpreted with caution. The study suffers from small sample size and lack of randomization, and there may be error from confounding factors from care seeking practices, patient's socio-economic status and/or selection/observer bias. With these limitations, the findings support the rationale for the use of RDTs in the management of children with malaria-pneumonia overlap. A randomized controlled trial that has accommodated these limitations is required to confirm these findings.

\section{ACKNOWLEDGEMENTS}

We wish to acknowledge the laboratory technicians and all caretakers of the children who participated in the study.

\section{REFERENCES}

1. Gove, S. Integrated management of childhood illness by outpatient health workers: technical basis and overview. The WHO Working Group on Guidelines for Integrated Management of the Sick Child. Bu 1l. World Health Organ. 1997; 75 (Suppl 1): 7-24.

2. Kallander K, Nsungwa-Sabiti J, Peterson S. Symptom overlap of malaria and pneumonia policy implications for home management strategies. Acta Tropica 2004; 90: 211-214.

3. World Health Organisation. Guidelines for the treatment of malaria, second edition. Geneva, WHO, 2010.

4. Ochola LB, Vounatsou P, Smith T, et al. The reliability of diagnostic techniques in the diagnosis and management of malaria in the absence of a gold standard. Lancet Infect Dis 2006; 6: 582-588.

5. Bisoffi Z, Sirima BS, Angheben A, et al. Rapid malaria diagnostic tests vs. clinical management of malaria in rural Burkina Faso: safety and outcome on clinical decisions. A randomized trial. Trop Med Int Health 2009; 14: 491 - 498.

6. Msellem MI, Martensson A, Rotllant G et al. Influence of Rapid Malaria Diagnostic Tests on Treatment and Health Outcome in Fever Patients, Zanzibar-A Crossover Validation Study. PLoS Med 2009; 6: e 1000070.

7. D'Acremont V, Kahama-Maro J, Mtasiwa D, Lengeler C, Genton B. Withdrawing antimalarials in febrile children with a negative rapid diagnostic test is safe in a moderately endemic area of Tanzania (abstract 397). ASTMH 57th Annual Meeting; 7-11 December 2008; New Orleans, Louisiana, United States.

8. World Health Organization. Consultative Meeting to Review Evidence and Research Priorities in the Management of Acute Respiratory Infections (ARI.) Geneva, 29 September - 1 October 2003 (Meeting Report 2004). Geneva, WHO. WHO/FCH/CAH/04.2. 
9. D'Acremont V, Kilowoko M, Kyungu E, et al. Etiology of fever in children from urban and rural Tanzania (abstract MIM16671706). $5^{\text {th }}$ MIM PanAfrican Malaria Conference; 2-6 November 2009; Nairobi, Kenya.

10. Sayang C, Soula G, Tahar R, et al. Use of a histidine-rich protein 2-based rapid diagnostic test for malaria by health personnel during routine consultation of febrile outpatients in a peripheral health facility in Yaoundé, Cameroon. Am J Trop Med Hyg, 2009; 81: 343-347.
11. Adjuik M, Babiker A, International Artemisin in Study Group et al. Artesunate combinations for treatment of malaria: meta-analysis. Lancet, 2004; 363: 9-17.

12. Ansah EK, Bana-Narh S, Akanpigbiam S, et al. Rapid testing for malaria in settings where microscopy is available and peripheral clinics where only presumptive treatment is available: a randomized controlled trial in Ghana. BMJ, 2010; 340: c 930. 